\title{
Affective, Normative and Continuance: Predictors of Employees' Commitment of Large-Cap It Firms in Indian Context
}

\author{
Radha Yadav $^{1 *}\left(\mathbb{D}\right.$, Ashu Khanna², Ruby Sengar ${ }^{3}$, Sudipta Dasmohapatra ${ }^{4}$ \\ ${ }^{1}$ USB, Chandigarh University, Punjab, India \\ ${ }^{2}$ IIT Roorkee, Roorkee, India \\ ${ }^{3}$ Symbiosis Centre for Management Studies, Noida, India \\ ${ }^{4}$ Duke University, Durham, USA \\ Email: *yadavradhika121@gmail.com
}

How to cite this paper: Yadav, R., Khanna, A., Sengar, R. and Dasmohapatra, S. (2019) Affective, Normative and Continuance: Predictors of Employees' Commitment of Large-Cap It Firms in Indian Context. Theoretical Economics Letters, 9, 1772-1791. https://doi.org/10.4236/tel.2019.96113

Received: May 8, 2019

Accepted: August 5, 2019

Published: August 8, 2019

Copyright (c) 2019 by author(s) and Scientific Research Publishing Inc. This work is licensed under the Creative Commons Attribution International License (CC BY 4.0).

http://creativecommons.org/licenses/by/4.0/

\begin{abstract}
Purpose: The purpose of the paper is to investigate the predictors of employees' commitment and also find out the critical dimensions of quality of work life (QWL) that engender commitment among in today's employees in information technology (IT) firms. Further, the study analyses on the association of demographic variables with QWL and organizational commitment (OC). Design/Methodology/Approach: The sample size for the study consists of 618 employees (respondents) from 21 large-caps (large-capital) IT companies in India. Cronbach alpha to test the reliability and validity; Factor Analysis as a data reduction tool. Subsequently, the mixed model and multi-regression methods are applied to test the relationship between the said variables. Findings: The findings suggest that 13 variables of QWL play a key role in the work life of IT employees. The results show that there is a significant relationship between the qualification and number of dependents with QWL and OC both. Moreover, a substantial relationship between QWL and affective commitment of IT employees is found. The results further reveal that there exists a strong link between various dimensions of QWL with OC. Practical Implications: QWL will help in creating a healthy environment in the organization that will enhance the commitment of the employees towards their organization. Enhanced OC will stimulate individual's attachment to the organization. Moreover, if QWL and OC are boosted, they will motivate employees to stay with the organization and enthusiastically work towards organizational objectives. Further, the escalation of OC will help in achieving growth, profit and market share at a greater pace. Originality/Value: This study focuses on the important variables for employees commitment in the terms of QWL.
\end{abstract}




\section{Keywords}

Demographic Characteristics, Quality of Work Life, Organizational Commitment, Affective Commitment, Continuous Commitment, and Normative Commitment

\section{Introduction}

With the world changing rapidly, managing an organization has become an increasingly complex act for the sustainable future and growth. The study provides an insight on how organizations develop and make changes in the QWL to get committed employees. Manager's work on those variables which are significantly related to QWL and OC and these variables are also useful for the sustainable growth [1]. The concept of QWL came into the limelight after the results of Hawthorne experiments (1924-1933) conducted by Elton Mayo and Fritz Roethlisberger [2]. Walton (1998) was the first person who introduced the concept of QWL having eight dimensions namely, fair payment, legal positivism, permanent growth and security, opportunity, social dependency, development of individual capabilities, security of environment and social integration [3]. The continuously revised policies and programs like performance appraisal, career growth, work-life balance, participative management, etc. created a positive impact on the morale of the employees [4] [5]. QWL is exhibited when the employee's incentive in the form of monetary and non-monetary services are provided by the organization [6].

The concept of organizational commitment (OC) has been derived as a concept from industrial and organizational psychology [7]. Commitment changes from individual to individual concerning attachments, opportunities accessibility, monetary advantages and so on [8] [9]. Porter et al. (1982) gave a "side-bet" theory which explains $\mathrm{OC}$ as the behavior involving individuals to the process in which they lock themselves in a particular organization [10] [11]. The idea of OC is depicted as a tridimensional idea, portrayed by the affective, continuance and normative measurements [12] [13].

\section{Purpose of the Study}

IT sector is an emerging and continuously blooming sector. According to the NASSCOM report 2012 \& Indian Times report 2017, IT sector has been continued to emerge as the prime engine of economic growth and contributing to nearly $13 \%$ of the Indian gross domestic product (GDP). IT sector gives the employment directly about 2.5 million people in India, so it is necessary to assess the quality of work life that enhance the organizational commitment i.e. affective, continuance and normative [14]. IT sector has been facing ups and downs for the last 10 years, with the employees' facing many problems like layoff, termination, decrement in salary, etc. With the advancement of technology, organizations began putting resources into the change of the work environment, attempting to make it appropriate to the physical, mental, and social needs of its 
employees, as this approach to forces its differential face in the business sector [15]. The purpose of this study is to analyze the predictors of employees' commitment with the respect for Quality of Worklife of the IT sector in the Indian context. Quality of Worklife is analyzed by investigating the dimensions that boost their performance, and to offer suggestions to improve their work-life balance.

\section{Objectives of the Study}

The aim of this study is to deliver the indicators based information that will enhance the performance of IT firms through exploring and assessing dimensions of QWL and predictors' employees' commitment. The study has been designed to attain the following objectives.

1) To understand the association of demographic variables of employees' with quality of work life and commitment.

2) To understand the association of dimensions of quality of work life and organizational commitment.

3) To understand the predictors of affective normative and continuance commitment.

\section{Literature Review}

\subsection{Dimensions of QWL and OC}

QWL, as an aspect, has evolved and affected a multitude of segments such as economic, technological and social era worldwide. To develop OC in employees, QWL should grow in companies. Past studies prove that QWL plays a vital role in generating the employee's willingness to stay in an organization and cultivates a positive attitude towards the job and organization. OC is a psychological commitment of employees where employees attach themselves to the organization with long-term loyalty [16]. The organization provides employees with job security, promotion opportunities, and better training and development and in return earn their loyalty, i.e., OC for long-term [17] [18]. Various dimensions of QWL such as fringe benefits, peer and superior relations, training and development, grievance handling procedures, etc., improve the satisfaction level of employees and generate commitment amongst them [19]. Studies prove that QWL plays a significant role in enhancing job satisfaction and organizational performance [20] [21]. Results from the previous analysis have shown that supervision, remuneration, and welfare schemes are positively correlated with affective, nominative and continuance commitment [17] [22] [23]. Previous result also shows that QWL is positively and significantly associated with OC which shows that OC is an outcome of better QWL [24]. Employee's satisfaction and commitment rely on the quality of work which they are receiving from the organization [21] [25] [26]. Fair and sufficient payment in the form of salary and allowances are positively correlated with the OC [23] [27]. It is also found that the essential variables for OC, which directly affects OC, are fair pay, health, safety and 
work conditions [17] [24]. One of the studies has found that QWL affects job embeddedness and commitment. There is a positive relationship between QWL and job embeddedness and affection commitment [28] [29]. It is a crucial point to create a healthy work-life for the employees. Healthy work-life improves the work satisfaction, reduce turnover and enhance productivity [30] [31].

\subsection{Association of QWL and OC}

Researcher tried to identify various dimensions of QWL and their degree of influence towards OC. Past studies proves that there is significant relationship with the QWL and OC [17] [31] [32] [33] [34] [35]. The null hypothesis opted for these variables in the study are as follows:

$H_{01}$ : There is no significant relation between QWL and OC;

$H_{01 a}$ : There is no significant relation between dimensions of QWL and affective commitment;

$H_{01 b}$ : There is no significant relation between dimensions of QWL and continuance commitment;

$H_{01 c}$ : There is no significant relation between dimensions of QWL and normative commitment.

\subsection{Demographic Variables with QWL and OC}

Past research stated that supervision, remuneration and welfare schemes are positively correlated with affective, nominative and continuance (alternative) commitment [33] [17] [23]. There is positive association between salary and allowances with commitment. Salary is associated with the qualification of the individual, it means that education and job experience are the main variables which describe the individual's commitment [36]. A study has proved that QWL is the requirement of the employees; it is not based on gender. Better QWL of the employees is necessary for all the employees whether he is male or a female [37] [38] [39] [40] [41]. Another study is done 292 IT employees which say that there is no relation of gender with the QWL but there is positive relation of age, work experience and income with the QWL [42].

Based on the literature, it can be said that dimensions of QWL help in developing employee's attachment towards the organization which propels the growth of an organization. After discussing literature review and noting identification of objectives following hypotheses were developed:

Association of demographic variables with $Q W L$ and $O C$

Demographic variable is an important factor that influences perception of individual's towards QWL in the organization and build the commitment among employees. Past studies have proven that marital status, designation, gender and experience have a significant relationship with the QWL and OC [1] [36]. The null hypothesis opted for this variable in the study as:

$H_{02}$ : There is no significant relation between demographic variables of employees with QWL. 
$H_{03}$ : There is no significant relation between demographic variables of employees with OC.

\section{Research Methodology}

\section{Sampling}

A total of 618 samples are collected from employees working with the IT Firms in India. The total number of large-cap (large-capital) IT firms in India is 30 approximately. The authors have approached all the large-cap IT firms, but data could only be collected from 21 companies. Data are collected through systematic random sampling. The questionnaire is developed by the self-administered questionnaire development process [13]. After the collection of 618 questionnaires with 65 items, the questionnaire is analyzed by using factor analysis in which principal component analysis, commonalities and rotated component matrix is used [43].

\section{Results and Analysis}

\subsection{Response Rate}

For the survey, 1000 questionnaires are distributed amongst the respondents of which 700 filled questionnaires came back. However, from the returned questionnaires, 82 questionnaires are discarded as they are filled partially, and are not adequate for the analysis. The actual response rate is $70 \%$. The sample consists of 618 questionnaires wholly filled by the IT sector employees.

\subsection{Demographic \& Dummy Variables}

In the study, the authors have chosen demographic variables such as gender, marital status, age, work experience, educational qualification, remuneration, designation, jobs changed, number of dependents and the spouse's income of the employees in IT firms. All these qualitative variables play an important role in the performance of the employees. The frequency distribution of the demographics is shown in Table 1. These qualitative variables are essentially nominal scale variables with no particular numerical values. So, firstly these variables are quantified by creating dummy variables, which takes values of 0 and 1.0 indicates the absence of an attribute and 1 indicates its presence. In the study, the following dummy variables will be used for multiple regressions. Variables are quantified as follows: gender (male: 1, female: 0 ); Age (below 35 years: 1, above 35 years: 0); marital status (married: 1, unmarried: 0 ); educational qualification (graduate: 1, postgraduate: 0 ); remuneration (below 5 lakhs: 1 , above 5 lakhs:0); work experience (Below 5 years: 1, above 5 years: 0 ); jobs changed (less than 2 jobs: 1 , more than 2 jobs: 0 ); dependents (no dependents: 1,1 or more dependents: 0 ) and spouse income (below 2.5 lakhs: 1 , above 2.5 lakhs: 0 ).

\subsection{KMO and Bartlett's Test of Sphericity}

The results of KMO and Bartlett's test of Sphericity have been illustrated in Table 2. 
Table 1. Descriptive statistics of demographic variables.

\begin{tabular}{|c|c|c|c|}
\hline Category & Sub-Category & No. of Respondents & Percentage \\
\hline \multirow{2}{*}{ Gender } & Male & 463 & 74.9 \\
\hline & Female & 155 & 25 \\
\hline \multirow{2}{*}{ Age } & Below 35 years & 459 & 74.2 \\
\hline & $35 \&$ above & 159 & 25.7 \\
\hline \multirow{2}{*}{ Marital status } & Married & 208 & 33.6 \\
\hline & Unmarried & 410 & 66.3 \\
\hline \multirow{2}{*}{ Educational Qualification } & Graduate & 318 & 51.5 \\
\hline & Post graduate & 300 & 48.5 \\
\hline \multirow{2}{*}{ Remuneration } & Below 5 lakhs & 361 & 58.4 \\
\hline & Above 5 lakhs & 257 & 41.6 \\
\hline \multirow{6}{*}{ Designation } & Manager & 34 & 5.5 \\
\hline & Executive & 103 & 16.6 \\
\hline & Engineer & 183 & 29.6 \\
\hline & Application developer & 133 & 21.5 \\
\hline & Trainee & 106 & 17.1 \\
\hline & Team Leader & 59 & 9.5 \\
\hline \multirow{2}{*}{ Work experience } & Below 5 years & 321 & 51.9 \\
\hline & Above 5 years & 297 & 48.1 \\
\hline \multirow{2}{*}{ Job changed } & Less than 2 jobs & 402 & 65.05 \\
\hline & More than 2 jobs & 216 & 34.95 \\
\hline \multirow{2}{*}{ Dependents } & No dependents & 444 & 71.85 \\
\hline & Dependents & 174 & 28.15 \\
\hline \multirow{2}{*}{ Spouse's Income } & Below 2.51 & 536 & 86.73 \\
\hline & Above 2.51 & 82 & 13.26 \\
\hline Total & & 618 & 100 \\
\hline
\end{tabular}

Table 2. KMO and Bartlett's test of sphericity.

\begin{tabular}{ccc}
\hline \multicolumn{2}{c}{ KMO and Bartlett's Test } & \\
\hline Kaiser-Meyer-Olkin Measure of Sampling Adequacy & & 0.798 \\
& Chi-Square & 8683.544 \\
Bartlett's test of Sphericity & df & 1653 \\
& Sig. & 0.000 \\
\hline
\end{tabular}

The results demonstrate that the value of KMO is 0.798 , which measures the sampling adequacy for conducting the study. Also, Bartlett's test of Sphericity is significant $(\mathrm{p}<0.001)$ here, which reveals the appropriateness of data for performing the factor analysis.

\subsection{Exploratory Factor Extraction Analysis of QWL's Variables}

Exploratory factor analysis (EFA) and Confirmatory factor analysis (CFA) are 
used to perform the extraction of variables. The test extracted 13 factors with 44 items. $57 \%$ of the variance is explained by the 13 factors along the condition of the Eigenvalue.

\subsection{Reliability}

The value of Cronbach's alpha of total item is 0.854 , which indicates a high level of internal consistency. This established adequate reliability with Cronbach alpha value greater than 0.70 [44] (Table 3).

It is found that 13 dimensions of QWL with 55 items cover all the aspects of the workplace. The details of the variables are given in Table 4, explored via factor loading and rotated component matrix during factor analysis (Figure 1).

\subsection{General Linear Model}

The possibility of the ordinary linear square model may not be appropriate, stems

Table 3. Reliability statistics of the total items and variables.

\begin{tabular}{ccc}
\hline \multicolumn{3}{c}{ Reliability Statistics } \\
\hline Cronbach's Alpha & Cronbach's Alpha Based on Standardized Items & No. of Items \\
\hline 0.854 & 0.845 & 55 \\
\hline
\end{tabular}

Table 4. Details of the items after factor analysis with reliability.

\begin{tabular}{|c|c|c|c|c|}
\hline Variables & & $\begin{array}{c}\text { Cronbach's Alpha } \\
\text { Based on } \\
\text { Standardized Items }\end{array}$ & $\begin{array}{c}\text { Before Factor } \\
\text { Analysis }\end{array}$ & $\begin{array}{c}\text { After Factor } \\
\text { Analysis }\end{array}$ \\
\hline Job security (JS) & 0.814 & 0.809 & 5 & 3 \\
\hline Participative management (PM) & 0.720 & 0.754 & 7 & 3 \\
\hline Peer relationship (PR) & 0.755 & 0.753 & 4 & 4 \\
\hline Superior relationship (PS) & 0.774 & 0.778 & 3 & 3 \\
\hline Work load (WL) & 0.729 & 0.727 & 4 & 3 \\
\hline Safety and harassment (SH) & 0.623 & 0.622 & 5 & 3 \\
\hline Rewards and recognition (RR) & 0.618 & 0.621 & 3 & 3 \\
\hline Career growth (CPD) & 0.701 & 0.702 & 3 & 5 \\
\hline Communication (C) & 0.718 & 0.721 & 3 & 3 \\
\hline Time pressure (TP) & 0.729 & 0.727 & 5 & 3 \\
\hline Pay (P) & 0.750 & 0.751 & 3 & 3 \\
\hline Work life balance (WLB) & 0.760 & 0.760 & 9 & 7 \\
\hline Fringe benefits (FB) & 0.765 & 0.765 & 12 & 12 \\
\hline Affective commitment (AC) & 0.702 & 0.704 & 4 & 4 \\
\hline Continuance commitment (CC) & 0.688 & 0.688 & 4 & 3 \\
\hline Normative commitment (NC) & 0.740 & 0.737 & 5 & 5 \\
\hline Total & & & 65 & 55 \\
\hline
\end{tabular}




\begin{tabular}{|c|c|c|c|c|c|c|c|c|c|c|c|c|c|}
\hline \multirow[b]{3}{*}{ FDD } & \multicolumn{13}{|c|}{ Component } \\
\hline & 1 & 2 & 3 & 4 & 5 & 6 & 7 & 8 & 9 & 10 & 11 & 12 & 13 \\
\hline & .820 & .029 & .093 & .027 & .418 & .055 & .013 & .034 & .069 & -.118 & -.099 & .163 & -.047 \\
\hline PP2 & 01 & .026 & .070 & .023 & .099 & .256 & .000 & -.057 & .040 & -.058 & -.251 & .057 & .087 \\
\hline DP3 & .710 & -.021 & .008 & -.049 & .159 & .176 & 147 & -.278 & .058 & .181 & -.020 & . 100 & .255 \\
\hline DP4 & .578 & .053 & .084 & .152 & .056 & -.149 & -.023 & -.073 & -.081 & .171 & .225 & .269 & .042 \\
\hline DP5 & .663 & .077 & -.062 & .132 & .226 & -.051 & -.007 & .041 & -.011 & .243 & .214 & .052 & -.103 \\
\hline LB1 & -.067 & .558 & -.005 & -.219 & .085 & .237 & .030 & .151 & .063 & -.008 & .200 & .023 & -.070 \\
\hline LB2 & $\mid-.155$ & .677 & -.061 & .049 & .076 & .086 & .161 & .075 & .115 & .110 & .263 & -.098 & -.055 \\
\hline & $\mid-.086$ & .614 & -.099 & .059 & .026 & .035 & 104 & -.042 & .064 & .253 & .065 & -.200 & .085 \\
\hline B4 & .092 & .658 & .155 & -.031 & -.030 & -.023 & .023 & -.122 & -.085 & .044 & -.106 & -.088 & .117 \\
\hline B5 & .097 & .642 & .149 & -.031 & -.034 & -.089 & -.125 & -.023 & -.093 & -.080 & -.081 & .146 & -.028 \\
\hline 6 & .185 & .643 & .041 & .042 & -.113 & -.176 & -.085 & -.050 & -.028 & -.096 & -.131 & .179 & .09 \\
\hline B7 & .093 & .597 & -.015 & .045 & -.001 & -.068 & -.046 & .095 & .069 & -.159 & -.057 & .009 & -.05 \\
\hline 1 & 93 & -.026 & .613 & .081 & .042 & .084 & -.016 & -.093 & .020 & .058 & .070 & .204 & .128 \\
\hline 2 & 02 & .009 & .711 & .042 & -.015 & .322 & -.083 & -.099 & .054 & -.034 & .196 & .141 & -.011 \\
\hline 3 & 17 & .066 & .814 & .081 & .040 & .153 & .030 & .047 & -.011 & .123 & .023 & .036 & .046 \\
\hline 4 & 21 & .061 & .745 & .213 & .049 & -.035 & .114 & .045 & .089 & .131 & -.102 & -.072 & 065 \\
\hline M1 & .016 & .025 & .166 & .770 & .085 & .086 & -.011 & -.087 & .026 & -.058 & .107 & .086 & .059 \\
\hline M2 & .155 & -.051 & .098 & .762 & -.010 & .117 & .050 & .032 & .019 & .006 & .020 & .012 & -.037 \\
\hline M3 & .226 & -.015 & .182 & .528 & .107 & .415 & .040 & -.028 & -.015 & .058 & -.229 & .062 & -.053 \\
\hline$x$ & .194 & -.103 & -.081 & .018 & .685 & .254 & -.001 & .015 & .094 & .272 & -.168 & .204 & .172 \\
\hline $\mathrm{C} 2$ & .057 & -.020 & .093 & .124 & .802 & -.033 & .035 & .033 & .016 & .019 & .152 & -.003 & .012 \\
\hline C3 & .313 & .002 & .037 & -.069 & .586 & -.059 & -.020 & -.072 & .061 & .147 & .158 & .022 & .074 \\
\hline SR1 & .226 & -.015 & .182 & .528 & .107 & .715 & .040 & -.028 & -.015 & .058 & -.229 & .062 & -.053 \\
\hline 32 & .164 & -.076 & .075 & .217 & -.038 & .720 & -.040 & -.038 & .034 & .059 & -.029 & -.055 & .001 \\
\hline 23 & 93 &.- .026 & .305 & .081 & .042 & .613 & -.016 & -.093 & .020 & .058 & .070 & .204 & .128 \\
\hline & 82 & .012 & .036 & .047 & .026 & -.005 & .604 & .231 & -.076 & -.072 & .124 & -.042 & .125 \\
\hline & 12 & -.044 & .078 & -.007 & -.004 & -.111 & .767 & .077 & .050 & .066 & .023 & .047 & .023 \\
\hline & 20 & .024 & -.012 & .008 & .002 & .058 & .657 & .094 & -.006 & -.050 & -.076 & .060 & -.125 \\
\hline & 46 & -.002 & .002 & .041 & -.018 & -.017 & .209 & .683 & -.234 & .009 & .138 & .072 & .017 \\
\hline 2 & 02 & -.005 & .043 & -.079 & -.080 & -.056 & .216 & .738 & .045 & .067 & .027 & .057 & -.016 \\
\hline TP3 & .095 & .139 & .010 & -.021 & .168 & -.075 & .081 & .459 & .059 & .195 & -.286 & -.091 & .264 \\
\hline JS1 & .073 & .098 & .077 & .112 & -.076 & -.076 & .132 & -.179 & .644 & .127 & .069 & -.028 & .067 \\
\hline JS2 & $\mid-.049$ & -.033 & .035 & -.162 & .185 & .036 & -.086 & -.072 & .602 & -.182 & -.006 & .174 & .088 \\
\hline IS 3 & .102 & -.033 & .035 & .075 & .025 & 101 & -.069 & 125 & .740 & .126 & -.176 & -.019 & -.159 \\
\hline $\mathrm{S} 1$ & .091 & -.005 & .137 & -.010 & .166 & .011 & -.028 & .033 & -.023 & .735 & .069 & .183 & -.020 \\
\hline 32 & .161 & -.079 & .198 & -.049 & .056 & .129 & -.043 & .126 & .155 & .692 & -.144 & -.040 & .105 \\
\hline (20 & .146 & .096 & .010 & .132 & .353 & 207 & -.019 & -.201 & -.077 & .805 & -.130 & .209 & -.078 \\
\hline $\mathbf{P}$ & .445 & .076 & -.012 & .209 & .107 & .295 & -.051 & -.108 & -.018 & .080 & .785 & -.101 & -.060 \\
\hline 12 & 0 & .056 & .089 & .051 & .102 & .086 & .129 & -.201 & -.044 & .102 & .721 & .004 & -.035 \\
\hline P3 & .092 & -.048 & -.006 & .004 & .093 & -.048 & -.005 & 157 & -.055 & -.093 & .662 & -.055 & .047 \\
\hline RR1 & & -.010 & -.166 & .150 & .048 & .148 & 209 & -.074 & .129 & .096 & -.140 & .561 & .128 \\
\hline & .009 & -.003 & .081 & .021 & .095 & .012 & -.020 & .118 & .016 & .070 & .023 & .789 & -.052 \\
\hline RR3 & 038 & .099 & .094 & .268 & -.178 & .062 & -.077 & -.046 & .217 & .197 & .185 & .781 & .076 \\
\hline $\mathrm{AB}$ & .029 & .013 & .041 & -.166 & 109 & -.025 & .078 & .062 & -.13 & .00 & -.06 & -.07 & .82 \\
\hline
\end{tabular}

(Note: JS: job security, PM: participative management, SR: superior relationship, PR: peer relationship, TP: time pressure, WL stands workload, SH: safety and harassment, RR: rewards and recognition, C: communication, CPD: career promotion and development, WLB: work-life balance, FB: fringe benefits).

Figure 1. Component analysis.

from the fact that, lumping together different companies may camouflage the heterogeneity (individual or uniqueness) that may exist among twenty-one companies. The difference may be due to unique features of a company such as human resource practices and policies. One way to take into account the heterogeneity that may exist among each company is to have its intercept.

Equation (1): 


$$
\begin{aligned}
\mathrm{QWL} / \mathrm{OC}= & \alpha+\beta_{1} \text { gender }+\beta_{2} \text { marital status }+\beta_{3} \text { age }+\beta_{4} \text { qualification } \\
& +\beta_{5} \text { work experience }+\beta_{6} \text { jobs changed }+\beta_{7} D_{1 i}+\beta_{8} D_{2 i} \\
& +\beta_{9} D_{3 i}+\cdots+\beta_{27} D_{20 i}+\varepsilon
\end{aligned}
$$

where,

$D_{1 i}, D_{2 i}, \cdots, D_{20 i}=$ for organization (such as 1 for organization 1, 0 otherwise; 1 for organization 2, 0 otherwise; and so on)

$\mathrm{QWL}=$ score of quality of work life $(\mathrm{QWL})$ and organizational commitment (OC)

$$
\begin{aligned}
& \alpha=\text { intercept } \\
& \beta_{1}, \beta_{2}, \cdots, \beta_{27}=\text { estimates of coefficient } \\
& \varepsilon=\text { error }
\end{aligned}
$$

This can be done quickly by introducing different intercept dummies for each company. This method is known as only fixed effect model. In this case, 20 dummies will represent differentiated intercept dummy coefficient. In the study, the authors are treating the first organization as a benchmark or reference category although any organization can be chosen for that purpose. If we examine organizations different intercept dummies, we find that several of them are statistically highly significant, indicating heterogeneity among 21 companies. The model is known as a one-way fixed-effect model, which allows intercepting to differentiate between cross-sectional data.

\subsection{Relationship of Demographic Variables with OC}

The following hypothesis is developed:

$\mathrm{H}_{01}$ : There is no significant relationship between demographic variables of employees and their perception towards OC.

Table 5 shows the result of multivariate regression of the demographic variables on the dependent variables. In this model, various organizations are used as a fixed factor, OC is used as dependent variable and dummy of demographic variables are used as a covariate.

\subsection{Relationship of Demographic Variables with QWL}

The following hypothesis is developed (Table 6):

$\mathrm{H}_{02}$ : There is no significant relationship between demographic variables of employees and their perception towards QWL.

\subsection{Regression Analysis}

Linear regression model is used to find the effect of dimensions of QWL on overall commitment and predictors of various types of commitment. Three types of linear regression models are used in this study which is as follows (Table 7).

Equation (2):

Model 1: $\mathrm{OC}=\alpha+\beta_{1} \mathrm{JS}+\beta_{2} \mathrm{PM}+\beta_{3} \mathrm{SR}+\beta_{4} \mathrm{PR}+\beta_{5} \mathrm{TP}+\beta_{6} \mathrm{WL}+\beta_{7} \mathrm{SH}$

$$
+\beta_{8} \mathrm{RR}-\beta_{9} \mathrm{C}+\beta_{10} \mathrm{CPD}+\beta_{11} \mathrm{P}+\beta_{12} \mathrm{WLB}+\beta_{13} \mathrm{FB}+\varepsilon
$$

Equation model for Affective Commitment 
Table 5. Tests of fixed effects for the demographic variables with organizational commitment.

\begin{tabular}{|c|c|c|c|c|c|c|c|}
\hline \multicolumn{8}{|c|}{ Parameter Estimates } \\
\hline Dependent Variable & & & & Norm & zed & & \\
\hline \multirow{3}{*}{ Parameter } & \multirow{3}{*}{ B } & \multirow{3}{*}{ Std. Error } & \multirow{3}{*}{$\mathrm{T}$} & \multirow{3}{*}{ Sig. } & \multicolumn{2}{|c|}{$\begin{array}{l}\text { 95\% Confidence } \\
\text { Interval }\end{array}$} & \multirow{3}{*}{$\begin{array}{l}\text { Partial Et } \\
\text { Squared }\end{array}$} \\
\hline & & & & & Lower & Upper & \\
\hline & & & & & Bound & Bound & \\
\hline Intercept & 57.591 & 2.803 & 20.547 & 0.000 & 52.086 & 63.096 & 0.417 \\
\hline Dummy gender & -0.697 & 0.928 & -0.751 & 0.453 & -2.518 & 1.125 & 0.001 \\
\hline Dummy age & -1.316 & 1.022 & -1.287 & 0.199 & -3.324 & 0.692 & 0.003 \\
\hline Dummy work experience & 0.530 & 0.922 & 0.575 & 0.565 & -1.280 & 2.341 & 0.001 \\
\hline Dummy qualification & 2.395 & 0.871 & 2.748 & 0.006 & 0.683 & 4.106 & 0.013 \\
\hline Dummy jobs changed & 1.169 & 0.965 & 1.211 & 0.226 & -.727 & 3.064 & 0.002 \\
\hline Dummy marital status & -1.068 & 0.968 & -1.103 & 0.270 & -2.969 & 0.833 & 0.002 \\
\hline Dummy dependents & -1.346 & 0.851 & -1.581 & 0.014 & -3.018 & 0.326 & 0.000 \\
\hline [org. 1] & 4.397 & 3.149 & 1.396 & 0.163 & -1.787 & 10.582 & 0.003 \\
\hline [org. 2] & 5.051 & 3.042 & 1.661 & 0.097 & -.923 & 11.025 & 0.005 \\
\hline [org. 3] & 6.651 & 3.046 & 2.184 & 0.029 & 0.669 & 12.634 & 0.008 \\
\hline [org. 4] & 4.245 & 3.377 & 1.257 & 0.209 & -2.387 & 10.878 & 0.003 \\
\hline [org. 5] & 5.755 & 3.239 & 2.777 & 0.016 & -.607 & 12.117 & 0.005 \\
\hline [org. 6] & 3.926 & 3.034 & 1.294 & 0.046 & -2.034 & 9.885 & 0.003 \\
\hline [org. 7] & -1.271 & 3.148 & -0.404 & 0.687 & -7.453 & 4.912 & 0.000 \\
\hline [org. 8] & 0.514 & 3.199 & 0.161 & 0.872 & -5.768 & 6.797 & 0.000 \\
\hline [org. 9] & 1.939 & 3.171 & 0.612 & 0.541 & -4.288 & 8.166 & 0.001 \\
\hline [org. 10] & 3.891 & 3.310 & 1.175 & 0.240 & -2.610 & 10.392 & 0.002 \\
\hline [org. 11] & 1.152 & 3.277 & 0.351 & 0.725 & -5.285 & 7.588 & 0.000 \\
\hline [org. 12] & 0.323 & 3.089 & 0.104 & 0.917 & -5.745 & 6.390 & 0.000 \\
\hline [org. 13] & 1.495 & 3.088 & 0.484 & 0.628 & -4.570 & 7.560 & 0.000 \\
\hline [org. 14] & 4.023 & 3.188 & 1.262 & 0.207 & -2.238 & 10.284 & 0.003 \\
\hline [org. 15] & 0.112 & 3.154 & 0.036 & 0.972 & -6.082 & 6.307 & 0.000 \\
\hline [org. 16] & -9.246 & 3.163 & -2.923 & 0.004 & -15.458 & -3.034 & 0.014 \\
\hline [org. 17] & -8.287 & 3.203 & -2.587 & 0.010 & -14.578 & -1.996 & 0.011 \\
\hline [org. 18] & -0.492 & 3.065 & -0.161 & 0.872 & -6.511 & 5.527 & 0.000 \\
\hline [org. 19] & 1.393 & 3.103 & 0.449 & 0.654 & -4.701 & 7.488 & 0.000 \\
\hline [org. 20] & -0.647 & 3.230 & -0.200 & 0.841 & -6.990 & 5.696 & 0.000 \\
\hline [org. 21] & $0^{\mathrm{a}}$ & & & & & & \\
\hline
\end{tabular}

a. This parameter is set to zero because it is redundant (Where B is the coefficient, sig. Is the probability or level of significance, $\mathrm{t}$ is $\mathrm{t}$-statistics, Org. is organization). 
Table 6. Tests of fixed effects for the demographic variables with QWL.

\begin{tabular}{|c|c|c|c|c|c|c|c|}
\hline \multicolumn{8}{|c|}{ Parameter Estimates } \\
\hline Dependent Variable: & & & & L Norn & lized & & \\
\hline \multirow{3}{*}{ Parameter } & \multirow{3}{*}{ B } & \multirow{3}{*}{$\begin{array}{l}\text { Std. } \\
\text { Error }\end{array}$} & \multirow{3}{*}{$\mathrm{T}$} & \multirow{3}{*}{ Sig. } & \multicolumn{2}{|c|}{ 95\% Confidence } & \multirow{3}{*}{$\begin{array}{c}\text { Partial Eta } \\
\text { Squared }\end{array}$} \\
\hline & & & & & Lower & Upper & \\
\hline & & & & & Bound & Bound & \\
\hline Intercept & 56.133 & 1.857 & 30.225 & 0.000 & 52.485 & 59.780 & 0.607 \\
\hline Dummy gender & -0.258 & 0.615 & -0.420 & 0.675 & -1.465 & 0.949 & 0.000 \\
\hline Dummy age & -0.927 & 0.677 & -1.368 & 0.172 & -2.257 & 0.404 & 0.003 \\
\hline Dummy work experience & 0.878 & 0.611 & 1.438 & 0.114 & -0.321 & 2.078 & 0.003 \\
\hline Dummy qualification & 1.315 & 0.577 & 2.278 & 0.023 & 0.181 & 2.449 & 0.009 \\
\hline Dummy jobs changed & 0.202 & 0.565 & 0.358 & 0.721 & -0.907 & 1.311 & 0.000 \\
\hline Dummy marital status & 0.861 & 0.639 & 1.347 & 0.179 & -0.395 & 2.117 & 0.003 \\
\hline Dummy dependents & -1.368 & 0.562 & -2.433 & 0.050 & -2.472 & -0.264 & 0.010 \\
\hline [org. 1] & 3.690 & 2.086 & 1.769 & 0.077 & -0.408 & 7.788 & 0.005 \\
\hline [org. 2] & 4.204 & 2.015 & 2.086 & 0.037 & 0.246 & 8.162 & 0.007 \\
\hline [org. 3] & 2.976 & 2.018 & 1.475 & 0.041 & -0.988 & 6.940 & 0.004 \\
\hline [org. 4] & 4.041 & 2.238 & 1.806 & 0.051 & -0.354 & 8.436 & 0.005 \\
\hline [org. 5] & 3.022 & 2.146 & 1.408 & 0.160 & -1.193 & 7.237 & 0.003 \\
\hline [org. 6] & 2.008 & 2.010 & 0.999 & 0.318 & -1.940 & 5.957 & 0.002 \\
\hline [org. 7] & 0.863 & 2.086 & 0.414 & 0.679 & -3.234 & 4.959 & 0.000 \\
\hline [org. 8] & -1.162 & 2.120 & -0.548 & 0.584 & -5.325 & 3.001 & 0.001 \\
\hline [org. 9] & 5.786 & 2.101 & 2.754 & 0.006 & 1.660 & 9.912 & 0.013 \\
\hline [org. 10] & 5.136 & 2.193 & 2.342 & 0.020 & 0.828 & 9.444 & 0.009 \\
\hline [org. 11] & 3.192 & 2.172 & 1.470 & 0.142 & -1.072 & 7.457 & 0.004 \\
\hline [org. 12] & 3.469 & 2.047 & 2.695 & 0.041 & -0.551 & 7.489 & 0.005 \\
\hline [org. 13] & 1.968 & 2.046 & 0.962 & 0.337 & -2.051 & 5.986 & 0.002 \\
\hline [org. 14] & 2.568 & 2.112 & 1.216 & 0.224 & -1.580 & 6.717 & 0.002 \\
\hline [org. 15] & 0.648 & 2.090 & 0.310 & 0.757 & -3.456 & 4.753 & 0.000 \\
\hline [org. 16] & -3.026 & 2.096 & -1.444 & 0.149 & -7.142 & 1.090 & 0.004 \\
\hline [org. 17] & -0.833 & 2.122 & -0.393 & 0.695 & -5.001 & 3.335 & 0.000 \\
\hline [org. 18] & 2.198 & 2.031 & 1.082 & 0.280 & -1.790 & 6.186 & 0.002 \\
\hline [org. 19] & 2.628 & 2.056 & 1.278 & 0.202 & -1.410 & 6.666 & 0.003 \\
\hline [org. 20] & 2.142 & 2.140 & 1.001 & 0.317 & -2.061 & 6.345 & 0.002 \\
\hline [org. 21] & $0^{\mathrm{a}}$ & & & & & & \\
\hline
\end{tabular}

a. This parameter is set to zero because it is redundant (Where B is the coefficient, sig. is the probability or level of significance, $t$ is $t$-statistics, Org. is organization). 
Table 7. Regression of dimensions of QWL on dependent variable OC.

\begin{tabular}{|c|c|c|c|}
\hline \multirow[t]{2}{*}{ Variables } & \multicolumn{3}{|c|}{$\begin{array}{l}\text { Model } 1 \\
\text { Dependent Variable: OC } \\
\text { Method: Linear Regression Method }\end{array}$} \\
\hline & Coefficients & $\mathrm{t}$ - stat & sig. \\
\hline (Constant) & 16.674 & 4.585 & 0.000 \\
\hline JS & 0.003 & 0.094 & 0.925 \\
\hline $\mathrm{PM}$ & 0.02 & 0.698 & 0.485 \\
\hline SR & 0.008 & 0.257 & 0.797 \\
\hline PR & 0.079 & 2.624 & 0.009 \\
\hline TP & 0.113 & 4.198 & 0.000 \\
\hline WL & 0.118 & 4.476 & 0.000 \\
\hline SH & 0.038 & 1.259 & 0.208 \\
\hline $\mathrm{RR}$ & 0.051 & 1.653 & 0.099 \\
\hline $\mathrm{C}$ & -0.026 & -0.898 & 0.370 \\
\hline $\mathrm{CPD}$ & 0.154 & 4.624 & 0.000 \\
\hline $\mathrm{P}$ & 0.094 & 3.435 & 0.001 \\
\hline WLB & 0.085 & 3.653 & 0.000 \\
\hline $\mathrm{FB}$ & 0.001 & -0.012 & 0.991 \\
\hline R-squared & 0.300 & & \\
\hline Adjusted R-squared & 0.285 & & \\
\hline Durbin-Watson stat & 1.912 & & \\
\hline F-statistics & 19.873 & & \\
\hline Significance ( F-stat) & 0.000 & & \\
\hline
\end{tabular}

a. Dependent variable: OC; b. Predictors: (constant), FB, SR, WLB, WL, JS, P, RR, SS, TP, C, PR, PM, CPD.

Model 1a: $\quad \mathrm{AC}=\alpha+\beta_{1} \mathrm{JS}+\beta_{2} \mathrm{PM}+\beta_{3} \mathrm{SR}+\beta_{4} \mathrm{PR}+\beta_{5} \mathrm{TP}+\beta_{6} \mathrm{WL}+\beta_{7} \mathrm{SH}$

$$
+\beta_{8} \mathrm{RR}-\beta_{9} \mathrm{C}+\beta_{10} \mathrm{CPD}+\beta_{11} \mathrm{P}+\beta_{12} \mathrm{WLB}+\beta_{13} \mathrm{FB}+\varepsilon
$$

Equation model for Continuance Commitment

$$
\text { Model 1b: } \quad+\beta_{8} \mathrm{RR}-\beta_{9} \mathrm{C}+\beta_{10} \mathrm{CPD}+\beta_{11} \mathrm{P}+\beta_{12} \mathrm{WLB}+\beta_{13} \mathrm{FB}+\varepsilon
$$

Equation model for Normative Commitment

Model 1c: $\quad \mathrm{NC}=\alpha+\beta_{1} \mathrm{JS}+\beta_{2} \mathrm{PM}+\beta_{3} \mathrm{SR}+\beta_{4} \mathrm{PR}+\beta_{5} \mathrm{TP}+\beta_{6} \mathrm{WL}+\beta_{7} \mathrm{SH}$

$$
+\beta_{8} \mathrm{RR}-\beta_{9} \mathrm{C}+\beta_{10} \mathrm{CPD}+\beta_{11} \mathrm{P}+\beta_{12} \mathrm{WLB}+\beta_{13} \mathrm{FB}+\varepsilon
$$

$H_{03}$ : There is no significant relation between dimensions of $Q W L$ and $O C$.

Equation (2). Model of $O C$

$$
\begin{aligned}
\mathrm{OC}= & 16.674+0.003 \mathrm{JS}+0.02 \mathrm{PM}+0.008 \mathrm{SR}+0.079 \mathrm{PR}+0.113 \mathrm{TP} \\
& +0.118 \mathrm{WL}+0.038 \mathrm{SH}+0.51 \mathrm{RR}-0.026 \mathrm{C}+0.154 \mathrm{CPD}+0.94 \mathrm{P} \\
& +0.085 \mathrm{WLB}+0.001 \mathrm{FB}+\varepsilon
\end{aligned}
$$

The following null hypotheses are taken for the analysis:

$H_{01 a}$ : There is no significant relation between dimensions of $Q W L$ and affective 
Table 8. Regression of dimensions of QWL with various types of OC.

\begin{tabular}{|c|c|c|c|c|c|c|c|c|c|}
\hline \multirow{2}{*}{ Variable } & \multicolumn{3}{|c|}{$\begin{array}{c}\text { Model 1a } \\
\text { Dependent Variable: AC }\end{array}$} & \multicolumn{3}{|c|}{$\begin{array}{c}\text { Model } 1 \mathrm{~b} \\
\text { Dependent Variable: CC }\end{array}$} & \multicolumn{3}{|c|}{$\begin{array}{c}\text { Model 1c } \\
\text { Dependent Variable: NC }\end{array}$} \\
\hline & coeff & $\mathrm{t}$ - stat & sig. & coeff & t- stat & sig. & coeff & t- stat & sig. \\
\hline Constant & 22.295 & 4.101 & 0.000 & 20.442 & 3.468 & 0.001 & -4.212 & -0.789 & 0.43 \\
\hline JS & 0.085 & 2.064 & 0.039 & -0.047 & -1.053 & 0.293 & -0.016 & -0.4 & 0.689 \\
\hline $\mathrm{PM}$ & 0.012 & 0.282 & 0.778 & 0.053 & 1.173 & 0.241 & -0.003 & -0.075 & 0.940 \\
\hline SR & 0.044 & 0.949 & 0.343 & -0.01 & -0.201 & 0.84 & -0.014 & -0.306 & 0.760 \\
\hline $\mathrm{PR}$ & -0.029 & -0.646 & 0.518 & 0.167 & 3.419 & 0.001 & 0.148 & 3.338 & 0.001 \\
\hline $\mathrm{TP}$ & 0.106 & 2.621 & 0.009 & 0.021 & 0.486 & 0.627 & 0.25 & 6.326 & 0.000 \\
\hline WL & 0.046 & 1.178 & 0.239 & 0.189 & 4.424 & 0.000 & 0.134 & 3.469 & 0.001 \\
\hline SS & 0.057 & 1.259 & 0.209 & -0.016 & -0.334 & 0.738 & 0.059 & 1.317 & 0.188 \\
\hline $\mathrm{RR}$ & 0.119 & 2.581 & 0.010 & 0.074 & 1.479 & 0.140 & -0.014 & -0.305 & 0.760 \\
\hline $\mathrm{C}$ & -0.04 & -0.934 & 0.351 & -0.067 & -1.434 & 0.152 & 0.019 & 0.454 & 0.650 \\
\hline $\mathrm{CPD}$ & 0.19 & 3.816 & 0.000 & 0.075 & 1.395 & 0.164 & 0.2 & 4.085 & 0.000 \\
\hline $\mathrm{P}$ & -0.001 & -0.026 & 0.98 & 0.139 & 3.14 & 0.002 & 0.167 & 4.162 & 0.000 \\
\hline WLB & 0.004 & 0.122 & 0.903 & 0.065 & 1.725 & 0.085 & 0.195 & 5.677 & 0.000 \\
\hline $\mathrm{FB}$ & 0.036 & 0.825 & 0.410 & 0.032 & 0.69 & 0.491 & -0.03 & -0.72 & 0.472 \\
\hline R-squared & & 0.126 & & & 0.148 & & & 0.336 & \\
\hline Adjusted R-squared & & 0.107 & & & 0.130 & & & 0.332 & \\
\hline Durbin-Watson & & 2.012 & & & 2.104 & & & 1.696 & \\
\hline F-stat. & & 6.681 & & & 8.086 & & & 23.51 & \\
\hline Sig. & & 0.000 & & & 0.000 & & & 0.000 & \\
\hline
\end{tabular}

Model $1 \mathrm{a}$ is the model of affective commitment, Model $1 \mathrm{~b}$ is continuance commitment and model $1 \mathrm{c}$ is normative commitment.

commitment.

$H_{01 b}:$ There is no significant relation between dimensions of $Q W L$ and continuance commitment.

$H_{01}$ : There is no significant relation between dimensions of $Q W L$ and normative commitment.

The effect of dimensions on affective, continuance and normative commitment are shown in Table 8 as shown above.

Equation 3.1. Model of affective commitment

$$
\mathrm{AC}=22.295+0.085 \mathrm{JS}+0.012 \mathrm{PM}+0.044 \mathrm{SR}-0.029 \mathrm{PR}+0.106 \mathrm{TP}
$$

Model 1a: $\quad+0.046 \mathrm{WL}+0.057 \mathrm{SH}+0.119 \mathrm{RR}-0.04 \mathrm{C}+0.19 \mathrm{CPD}-0.001 \mathrm{P}$

$$
+0.004 \mathrm{WLB}+0.036 \mathrm{FB}+\varepsilon
$$

In the first model, there is $10 \%$ variation among the dimensions of the QWL. Time pressure, career promotions and development, rewards and recognition and job security are highly significant with the affective commitment than the other aspects of the QWL.

Equation 3.2. Model of continuance commitment 


$$
\begin{aligned}
\mathrm{CC}= & 20.442-0.047 \mathrm{JS}+0.053 \mathrm{PM}-0.01 \mathrm{SR}+0.167 \mathrm{PR}+0.021 \mathrm{TP} \\
& +0.189 \mathrm{WL}+0.016 \mathrm{SH}+0.074 \mathrm{RR}-0.067 \mathrm{C}+0.075 \mathrm{CPD} \\
& +0.139 \mathrm{P}+0.065 \mathrm{WLB}+0.032 \mathrm{FB}+\varepsilon
\end{aligned}
$$

In the second model, there is $13 \%$ variation among the dimensions of the QWL. Peer relationship, workload, and career promotions and development are highly significant with the continuance commitment than the other dimensions of the QWL.

$$
\begin{aligned}
& \text { Equation 3.3. Model of normative commitment } \\
& \qquad \begin{aligned}
\mathrm{NC}= & -4.212-0.016 \mathrm{JS}-0.003 \mathrm{PM}-0.014 \mathrm{SR}+0.148 \mathrm{PR}+0.25 \mathrm{TP} \\
& +0.134 \mathrm{WL}+0.059 \mathrm{SH}-0.014 \mathrm{RR}+0.019 \mathrm{C}+0.2 \mathrm{CPD}+0.67 \mathrm{P} \\
& +0.195 \mathrm{WLB}-0.03 \mathrm{FB}+\varepsilon
\end{aligned}
\end{aligned}
$$

In the third model, there is 33\% variation among the dimensions of the QWL. Peer relationship, work load, time pressure, pay, work-life balance and career promotions and development are highly significant with the normative commitment than the other dimensions of the QWL. So, the value of F given in the test rejects the null hypothesis.

\section{Findings}

The findings of the study reveals that variables of quality of work life such as: career progress and development, peer and superior relationship, rewards and recognitions, work life balance, peer relationship, superior relationship and job security, fringe benefits, plays a vital role in the IT firms in the current scenario after checking the validity and reliability of the variables.

On application of the general linear model, it is found that there exists a relationship between demographic variables and OC. The results show that lesser educational qualification and presence of dependents have a significant positive relationship with OC. Gender, marital status, age group, work experience, jobs changed and work experience does not have any significant association with OC. The coefficient of gender and age group is -0.697 and -1.316 . It explains that average score of OC for a male employee is lower by about -0.697 units as compared to the average score of $\mathrm{OC}$ of female employees. In the case of age group, the average score of the OC for below 35 years of age employees is lower by -1.316 units as compared to the employees having age more than 35 years of age employees, but not significant. Employees who have 5-year work experience raise the average score of OC by 0.530 as compared to employees whose work experience is more than 5 years, but it is also not significantly correlated.

Similarly, it is found that there exists a positive association between demographic variables and QWL. The results show that educational qualification has a significant positive relationship with QWL. Gender, marital status, age group, dependent child, dependent adult, works experience do not have any significant association with the QWL [45]. The coefficient of gender and age group are -0.258 and -0.927 . It means that average score of QWL for a male employee is lower by about -0.258 units as compared to the average score of QWL of a fe- 
male employee. In the case of age group, the average score of QWL for the below 35 years of age employees is lower by -0.927 units as compared to the respondents having age more than 35 years, but both the variables are not significant. Employees having work experience of fewer than 5 years have an average score of QWL which is higher by 0.878 as compared to employees having work experience more than 5 years, but are not significantly correlated.

The results show that the adjusted $\mathrm{R}^{2}$ in the model is 0.285 which means that the linear regression explains $28.5 \%$ of the variance in the data. There are some predictors such as peer relationship, time pressure, workload, career promotion and development, pay and work-life balance which create high impact on OC. The results show that the value of $\mathrm{F}=19.873$ that explains there is a significant high association between the dependent and independent variables (predictors).

On performing the regression analysis between QWL and OC, it is found that there is a positive relationship between the two. The coefficient of peer relationship (0.79), time pressure (0.113), workload (0.118), career growth (0.154), pay (0.94) and work-life balance (0.85) are significant with OC. Time pressure, workload and career growth are the three predictors that play a vital role in the life of employees and develop an attachment towards the organization. Dimensions of QWL are found to be profoundly influencing the dependent variable OC and its aspects. Thus it can be seen in Table 7 that there is a relationship between predictors and dependent variables in this model. So, the value of $\mathrm{F}$ given in the test rejects the null hypothesis.

There is a significantly positive correlation between QWL and types of OC of employees. Time pressure, workload and career growth are essential three predictors, which play a vital role in the life of employees to create attachment towards the organization [46] [47]. The employee's commitment and decision to stay in the organization are guided by an emotional bond with the colleagues and the organization rather than by any logical reasoning [48].

This paper gives the important dimensions of QWL which creates commitment in today's IT employees towards the organization. In the end, it can be seen that job satisfaction, rewards and recognition and career promotion and development are highly significant with the affective commitment at the level of $5 \%$ significance. Peer relationship, workload, and career promotions and development are positively substantial with the continuance commitment at the $2 \%$ significance level. Where, peer relationship, workload, time pressure, pay, work-life balance, and career promotions and development are highly significant with the normative commitment at the level of $1 \%$ significance.

\section{Practical Implication}

QWL has become the most crucial factor in every sector. QWL has been developed as one of the mandatory dimensions for the publically listed companies which should be followed to improve the employee's welfare and society at large. QWL will help in creating a healthy environment in the organization that will 
enhance the commitment of the employees towards their organization. Enhanced OC will stimulate individual's attachment to the organization. Moreover, if QWL and OC are boosted, they will motivate employees to stay with the organization and enthusiastically work towards organizational objectives. Further, the escalation of OC will help in achieving growth, profit and market share at a greater pace.

\section{Conclusions}

The role of demographic variables is essential in the lives of employees to establish their association with QWL and OC. The educational qualification also plays a vital role in forming their perception towards things. At a workplace, educational qualification of the employees is highly significant with QWL and OC, where higher and better-educated employees do not perceive better QWL as compared to lesser qualified employees. The reason behind this could be the fact that the highly skilled employees expect more from the organization. Responsibility and care of the family is the necessity of the individual. If an employee has more dependent members, then the commitment of an employee towards the organization will increase, and the perception towards organization will improve because the decision of switching from the current organization may disrupt the life of the dependents. The finding of the study identifies that the educational qualification and the number of dependents are significantly related to QWL and OC.

This paper has found a positive and significant relationship between the dimensions of QWL and types of OC on a sample of employees from IT sector. Time pressure, workload, and career growth are essential three predictors, which play a vital role in the life of employees to create attachment towards the organization [46]. This study gives the essential dimensions of QWL which creates commitment in today's IT employees towards their organization [41] [49].

If an organization provides low QWL to its employees, then employees will inevitably not get attached to the organization, thus increasing the rate of burnout and eventually will move away from the organization. It has been clear from the findings that impact of QWL through factors like career progress development, peer and superior relationship, participative management, rewards and recognition, work-life balance, fringe benefits, safety, peer relationship, superior relationship and job security can attract the commitment of the employees towards the organization. The terms like burnout, attrition, etc. directly affects the cost and environment of the organization, which is detrimental to the organization regarding growth and competition.

\section{Future Scope and Limitations}

QWL and OC affect the performance of the organization. The study fills the literature gap of QWL, OC and organizational performance. To get accurate information about the relationship between QWL, OC and organizational perfor- 
mance, other sectors of the industry should also be taken into consideration. The comparison should be done with other sectors or large-cap companies with medium capital (mid-cap) companies or small capital (small-cap) companies. The future studies should consider new variables that are not used in this study. The other variables that influence the perception of employees as well as affect QWL are technological changes, training, and development, grievances handling procedures, personality traits etc. The above said variables are yet to be researched to analyze QWL, OC and their impact on organizational performance.

There are always some shortcomings in the research work that cannot be removed. Though the researchers have taken due care in data collection, analysis, and interpretation but there are some shortcomings in the research. Firstly, both primary and secondary data sources are used in the study. Qualitative research is always time-consuming and quite expensive. Primary data collection method is the dominant method to get information directly from the employees, but it has some flaws as well. Sometimes employees do not feel comfortable with the researcher to share all the information related to them. Moreover, the views of the respondents in the study are the reflection of sample size chosen, but we cannot accurately say that it does not reflect the views of the universe. The interpretation and specification of QWL and OC that are empirically examined in the present study must be regarded as tentative.

\section{Conflicts of Interest}

The authors declare no conflicts of interest regarding the publication of this paper.

\section{References}

[1] Oshagbemi, T. (2000) Gender Differences in the Job Satisfaction of University Teachers. Women in Management Review, 15, 331-343. https://doi.org/10.1108/09649420010378133

[2] Roethlisberger, F.J. and Dickson, W.J. (2003) Management and the Worker. Psychology Press, East Sussex.

[3] Walton, R.E. (1973) Quality of working life: what is it. Sloan management review, 15, 11-21.

[4] Luthans, F. (2011) Organizational Behaviour: An Evidence-Based Approach. McGraw-Hill, New York.

[5] Nadler, D.A. and Lawler, E.E. (1983) Quality of Work Life: Perspectives and Directions. Organizational Dynamics, 11, 20-30. https://doi.org/10.1016/0090-2616(83)90003-7

[6] Mahadzirah, M., Abdul Manan, A., Nur Izzati, A.G. and Ahmad Rusdi, A. (2012) Malaysia's Tourism Industry Performance and Its Destination Image. Business Management Series, 3, 61-70.

[7] Baruch, Y. and Cohen, A. (2007) The Dynamics between Organisational Commitment and Professional Identity Formation at Work. In: Brown, A., Kirpal, S. and Rauner, F., Eds., Identities at Work. Technical and Vocational Education and Training. Issues, Concerns and Prospects, Springer, Dordrecht, 241-260. 
https://doi.org/10.1007/978-1-4020-4989-7_9

[8] Stanton, N. and Matthews, G. (1995) Twenty-One Traits of Personality: An Alternative Solution for the Occupational Personality Questionnaire. Journal of Management Development, 14, 66-75. https://doi.org/10.1108/02621719510097370

[9] Parvar, M.R.F., Allameh, S.M. and Ansari, R. (2013) Effect of Quality of Work Life on Organizational Commitment by SEM (Case Study: OICO Company). International Journal of Academic Research in Business and Social Sciences, 3, 135. https://doi.org/10.6007/IJARBSS/v3-i10/285

[10] Porter, L., Steers, R., Mowday, R. and Boulian, P. (1974) Organizational Commitment, Job Satisfaction and Turnover among Psychiatric Technicians. Journal of Applied Psychology, 59, 603-609. https://doi.org/10.1037/h0037335

[11] Mowday, R.T., Steers, R.M. and Porter, L.W. (1979) The Measurement of Organizational Commitment. Journal of Vocational Behavior, 14, 224-247. https://doi.org/10.1016/0001-8791(79)90072-1

[12] Meyer, J.P. and Allen, N.J. (1984) Testing the" Side-Bet Theory" of Organizational Commitment: Some Methodological Considerations. Journal of Applied Psychology, 69, 372-378. https://doi.org/10.1037/0021-9010.69.3.372

[13] Meyer, J.P. and Allen, N.J. (1991) A Three-Component Conceptualization of Organizational Commitment. Human Resource Management Review, 1, 61-89. https://doi.org/10.1016/1053-4822(91)90011-Z

[14] NASSCOM (2017) Information Technology/Business Process Management (IT-BPM) Sector in India as a Share of India's Gross Domestic Product (GDP) from 2009 to 2017.

[15] Rajalakshmi, N. (2017) It's Time for Ctrl+Alt+Delete. The Hindu.

[16] Argyris, C. (1973) Personality and Organization Theory Revisited. Administrative Science Quarterly, 18, 141-167. https://doi.org/10.2307/2392060

[17] Sajjad, N.K. and Abbasi, B. (2014) Studying the Relationship between Quality of Work Life and Organizational Commitment. Research Journal of Recent Sciences, 3, 92-99.

[18] Feldman, M.S. (2000) Organizational Routines as a Source of Continuous Change. Organization Science, 11, 611-629. https://doi.org/10.1287/orsc.11.6.611.12529

[19] Riley, D. (2006) Turnover Intentions: The Mediation Effects of Job Satisfaction, Affective Commitment and Continuance Commitment. Doctoral Dissertation, The University of Waikato, Hamilton.

[20] Lewis, D., Brazil, K., Krueger, P., Lohfeld, L. and Tjam, E. (2001) Extrinsic and Intrinsic Determinants of Quality of Work Life. Leadership in Health Services, 14, 9-15. https://doi.org/10.1108/13660750110391539

[21] Talebi, B., Pakdelbonab, M., Zemestani, G. and Aghdami, N. (2012) Investigation the Relationship between the Employee's Quality of Work Life (QWL) and Their Effectiveness in Banking. European Journal of Experimental Biology, 2, 1839-1842.

[22] Normala, D. (2010) Investigating the Relationship between Quality of Work Life and Organizational Commitment amongst Employees in Malaysian Firms. International Journal of Business and Management, 5, 75. https://doi.org/10.5539/ijbm.v5n10p75

[23] Farjad, H.R. and Varnous, S. (2013) Study of Relationship of Quality of Work Life (QWL) and Organizational Commitment. Interdisciplinary Journal of Contemporary Research in Business, 4, 449-456.

[24] Tabassum, A., Rahman, T. and Jahan, K. (2012) An Evaluation of the Quality of 
Work Life: A Study of the Faculty Members of Private Universities in Bangladesh. $A B A C$ Journal, 32, 36-57.

[25] Kara, D., Uysal, M., Sirgy, M.J. and Lee, G. (2013) The Effects of Leadership Style on Employee Well-Being in Hospitality. International Journal of Hospitality Management, 34, 9-18. https://doi.org/10.1016/j.ijhm.2013.02.001

[26] Arif, S. and Ilyas, M. (2013) Quality of Work-Life Model for Teachers of Private Universities in Pakistan. Quality Assurance in Education, 21, 282-298. https://doi.org/10.1108/QAE-Feb-2012-0006

[27] Jayakumar, A. and Kalaiselvi, K. (2012) Quality of Work Life: An Overview. International Journal of Marketing, Financial Services \& Management Research, 1, 140-151.

[28] Zhao, X., Sun, T., Cao, Q., Li, C., Duan, X., Fan, L. and Liu, Y. (2013) The Impact of Quality of Work Life on Job Embeddedness and Affective Commitment and Their Co-Effect on Turnover Intention of Nurses. Journal of Clinical Nursing, 22, 780-788.

[29] Martins, N. and Van der Berg, Y. (2013) The Relationship between Organisational Trust and Quality of Work Life. SA Journal of Human Resource Management, 11, 1-13. https://doi.org/10.4102/sajhrm.v11i1.392

[30] Almalki, M.J., Fitzgerald, G. and Clark, M. (2012) The Relationship between Quality of Work Life and Turnover Intention of Primary Health Care Nurses in Saudi Arabia. BMC Health Services Research, 12, 314. https://doi.org/10.1186/1472-6963-12-314

[31] Farid, H., Izadi, Z., Ismail, I.A. and Alipour, F. (2015) Relationship between Quality of Work Life and Organizational Commitment among Lecturers in a Malaysian Public Research University. The Social Science Journal, 52, 54-61. https://doi.org/10.1016/j.soscij.2014.09.003

[32] Nair, S. (2013) A Study on the Effect of Quality of Work Life (QWL) on Organizational Citizenship Behavior (OCB)-With Special Reference to College Teachers Is Thrissur District, Kerala. Integral Review-A Journal of Management, 6, 34-46.

[33] Normala, D. (2010) Investigating the Relationship between Quality of Work Life and Organizational Commitment amongst Employees in Malaysian Firms. International Journal of Business and Management, 5, 75-43. https://doi.org/10.5539/ijbm.v5n10p75

[34] Ojedokun, O., Idemudia, E.S. and Desouza, M. (2015) Perceived External Prestige as a Mediator between Quality of Work Life and Organisational Commitment of Public Sector Employees in Ghana. SA Journal of Industrial Psychology, 41, 1-10. https://doi.org/10.4102/sajip.v41i1.1216

[35] Risla, M.K.F. and Ithrees, A.G.I.M. (2018) The Impact of Quality of Work Life on Organizational Commitment with Special Reference to Department of Community based Corrections. Global Journal of Management and Business Research, 18.

[36] Indumathy, R. (2012) A Study on Quality of Work Life among Workers with Special Reference to Textile Industry in Tirupur District: A Textile Hub. Journal of Contemporary Management Research.

[37] Bellou, V. (2010) Organizational Culture as a Predictor of Job Satisfaction: The Role of Gender and Age. Career Development International, 15, 4-19. https://doi.org/10.1108/13620431011020862

[38] Marsden, P.V., Kalleberg, A.L. and Cook, C.R. (1993) Gender Differences in Organizational Commitment: Influences of Work Positions and Family Roles. Work and 
Occupations, 20, 368-390. https://doi.org/10.1177/0730888493020003005

[39] Lankau, M.J. and Scandura, T.A. (2002) An Investigation of Personal Learning in Mentoring Relationships: Content, Antecedents, and Consequences. Academy of Management Journal, 45, 779-790.

[40] Ganesh, S. and Paramasivam Ganesh, M. (2014) Effects of Masculinity-Femininity on Quality of Work Life: Understanding the Moderating Roles of Gender and Social Support. Gender in Management. An International Journal, 29, 229-253. https://doi.org/10.1108/GM-07-2013-0085

[41] Yadav, R. and Khanna, A. (2014) Literature Review on Quality of Work Life and Their Dimensions. IOSR Journal of Humanities and Social Science, 19, 71-80. https://doi.org/10.9790/0837-19957180

[42] Bolhari, A., Rezaeean, A., Bolhari, J., Bairamzadeh, S. and Soltan, A.A. (2011) The Relationship between Quality of Work Life and Demographic Characteristics of Information Technology Staffs. Proceedings of CSIT, 5, 374-378.

[43] Thompson, B. (2007) Factor Analysis. In: The Blackwell Encyclopedia of Sociology, John Wiley \& Sons, Hoboken, NJ. https://doi.org/10.1002/9781405165518.wbeosf003

[44] Santosh, P.J. and Baird, G. (1999) Psychopharmacotherapy in Children and Adults with Intellectual Disability. The Lancet, 354, 233-242. https://doi.org/10.1016/S0140-6736(98)07059-7

[45] Hrebiniak, L.G. and Alutto, J.A. (1972) Personal and Role-Related Factors in the Development of Organizational Commitment. Administrative Science Quarterly, 17, 555-573. https://doi.org/10.2307/2393833

[46] Taggar, S. and Parkinson, J. (2007) Personality Tests in Accounting Research. Journal of Human Resource Costing \& Accounting, 11, 122-151. https://doi.org/10.1108/14013380710778776

[47] Aluwihare-Samaranayake, D., Gellatly, I., Cummings, G. and Ogilvie, E.L. (2018) A Contextual Work-Life Experiences Model to Understand Nurse Commitment and Turnover. Journal of Advanced Nursing, 74, 2053-2063. https://doi.org/10.1111/jan.13718

[48] Pradhan, S. and Pradhan, R.K. (2015) An Empirical Investigation of Relationship among Transformational Leadership, Affective Organizational Commitment and Contextual Performance. Vision, 19, 227-235. https://doi.org/10.1177/0972262915597089

[49] Faizan, R. and Zehra, N. (2016) Quality Work-Life as Predictor to Organisational Commitment under Contrasting Leadership Styles: IT Responses from Pakistans Private Software Houses. Global Journal of Management and Business Research, 16, $9-23$. 